Check for updates

Cite this: RSC Adv., 2017, 7, 53385

\title{
Enhanced antioxidant activity, antibacterial activity and hypoglycemic effect of luteolin by complexation with manganese(II) and its inhibition kinetics on xanthine oxidase
}

\begin{abstract}
Hao Dong, $\mathbb{D} \dagger$ Xiaocui Yang, $\dagger$ Jiapeng He, $\uparrow$ Sheng Cai, $\uparrow$ Kaijun Xiao $\dagger^{*}$ and Liang Zhu†
The present study aims to improve the biological activities of luteolin by complexation with manganese(॥). UV-visible spectroscopy, infrared spectroscopy, thermogravimetric analysis and elemental analysis were adopted to assess the relevant interaction of luteolin and manganese(॥) ions and the chelation sites. The antioxidant activity, hypoglycemic effect and antimicrobial activity of luteolin-manganese(॥) complex with respect to its parent luteolin and the inhibition effect of which on xanthine oxidase were investigated and compared. The spectroscopic data indicated that luteolin reacts with manganese(॥) cations through the chelation sites of 5-hydroxy and 4-carbonyl in two luteolin molecules. Antioxidant and antibacterial activity were enhanced after the complexation of manganese(॥) cations with luteolin. An inhibition effect assay found that luteolin and luteolin-manganese(॥) complex reversibly inhibited xanthine oxidase in a competitive manner. Luteolin-manganese(॥) complex had a more remarkable hypoglycemic effect than luteolin by increasing the glucose consumption in liver tissue.
\end{abstract}

Received 7th October 2017

Accepted 13th November 2017

DOI: $10.1039 / \mathrm{c} 7 \mathrm{ra11036g}$

rsc.li/rsc-advances flavonoids like quercetin, rutin and luteolin with a large number of metal cations such as $\mathrm{Cu}(\mathrm{II}),{ }^{18} \mathrm{Zn}(\mathrm{II}),{ }^{11} \mathrm{~V}$ (II) $,{ }^{15} \mathrm{Mo}(\mathrm{VI}),{ }^{19}$ $\mathrm{Fe}(\mathrm{II}),{ }^{20} \mathrm{Fe}(\mathrm{III}),{ }^{12} \mathrm{Sn}(\mathrm{II}){ }^{14}$ and $\mathrm{Cr}(\mathrm{III})^{21}$ has already been reported. In a previous report, copper-quercetin complex was synthesized and confirmed to be much more effective in free radical scavenging activity than the free quercetin. ${ }^{18}$ The complex formation between luteolin and vanadium(Iv) oxide sulphate monohydrate was investigated in another work. ${ }^{17}$ It was observed that the free radical scavenging activity and ferric ion reducing potential of luteolin was increased after the formation of complex with vanadium oxide cation.

Despite increasing studies of various metal-flavonoids complex and their biological activities, ${ }^{11,15,17,18,21,22}$ research about the complexation between luteolin and manganese(II) cation has been limited. Therefore, the purpose of the present study was to examine the complex formation between luteolin and manganese(II) cation and confirm the complexation sites in luteolin by means of UV-visible spectroscopy, infrared spectroscopy, thermogravimetric analysis and elemental analysis. Moreover, the variation of antioxidant property, antibacterial activity and hypoglycemic effect of luteolin after chelation with manganese were investigated. In the meantime, the inhibition effects and inhibition kinetics of luteolin and its mental complex on xanthine oxidase were evaluated. Results obtained in this work are expected to provide significant clues to further exploration of luteolin-manganese(II) complex with enhanced biological activities as a more effective xanthine oxidase inhibitor.
School of Food Science and Technology, South China University of Technology, Guangzhou 510640, People's Republic of China. E-mail: dong.h@alu.scut.edu.cn; fekjxiao@scut.edu.cn; Fax:+86-20-87113843; Tel: +86-20-87113843

$\dagger$ Current address: No. 381, Wushan Road, Tianhe District, Guangzhou, People's Republic of China. 


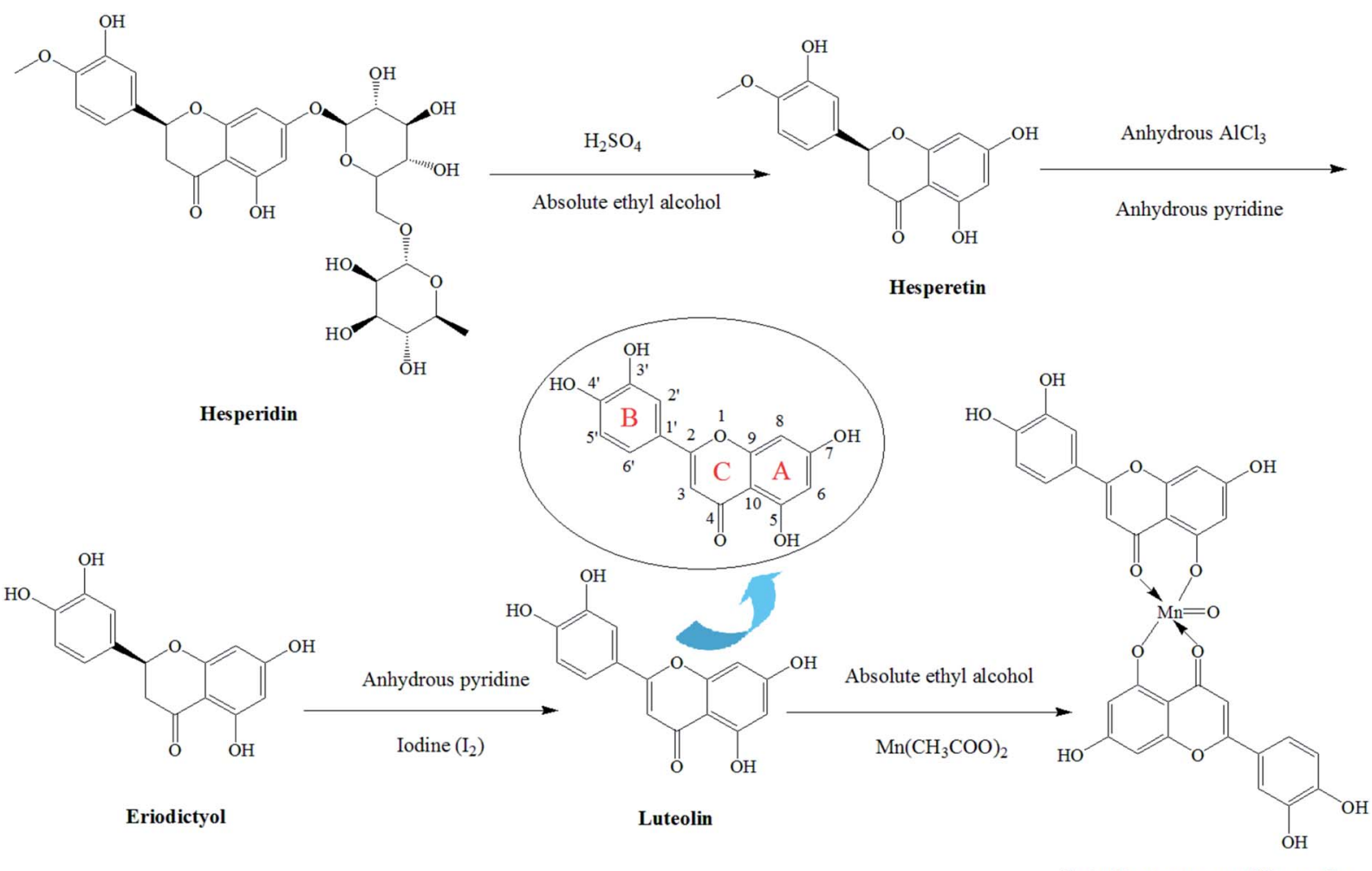

Luteolin-manganese(II) complex

Fig. 1 The proposed synthetic path of the co-ordination complex, luteolin-manganese(II) complex.

\section{Materials and methods}

\subsection{Materials and chemicals}

Hesperidin (pure: 99\%) was purchased from Xi'an Realin Biotechnology Co., Ltd. (Xi'an, China). Manganese acetate $\left(\mathrm{Mn}\left(\mathrm{CH}_{3} \mathrm{COO}\right)_{2}\right)$, 2,2-diphenyl-1-picrylhydrazyl (DPPH), xanthine and xanthine oxidase were purchased from Sigma Aldrich Chemical Co. (St. Louis, MO, USA). Escherichia coli, Staphylococcus aureus, Listeria monocytogenes and Pseudomonas aeruginosa were provided by Guangdong Culture Collection Center (Guangzhou, China). Luria-Bertani (LB) bouillon culture-medium and LB agar culture-medium were purchased from Guangdong Huankai Microbial Science and Technology Co. (Guangzhou, China). Human hepatoma (HepG2) cells (human hepatoma cell line) were purchased from American Type Culture Collection (Rockville, MD, USA). RPMI 1640 medium, Hanks' Balanced Salt Solution (HBSS), Dulbecco's modified eagle medium (DMEM), Fetal bovine serum (FBS), phosphate-buffered saline (PBS, pH 7.4), insulin, penicillin, streptomycin, gentamycin, sterile water and other cell culture reagents were purchased from Gibco Life Technologies Co. (Grand Island, NY, USA). All other chemicals of analytical grade were acquired from Guangzhou Chemical Reagent Factory (Guangzhou, China).

\subsection{Synthesis of luteolin}

Firstly, $10 \mathrm{~g}$ of hesperidin were weighed into a round-bottom flask and absolute ethyl alcohol $(300 \mathrm{~mL})$ was added to dissolve it. Then $10 \mathrm{~mL} \mathrm{H}_{2} \mathrm{SO}_{4}$ was added and the mixture was placed in a water-bath at $60{ }^{\circ} \mathrm{C}$ for complete reaction. Afterwards, reaction solution was extracted with ethyl acetate $(3 \times$ $300 \mathrm{~mL}$ ) and the organic phase was discarded. Deionized water and $5 \% \mathrm{NaCl}$ were orderly used to wash the solution. The hesperetin was obtained from the solution by vacuum drying.

The hesperetin ( $3.5 \mathrm{~g}$ ) was dissolved in anhydrous pyridine. Then anhydrous $\mathrm{AlCl}_{3}(1.5 \mathrm{~g})$ was added and the mixture was kept in a water-bath at $70{ }^{\circ} \mathrm{C}$ for complete reaction. After that, anhydrous pyridine was evaporated and the red residue was added with $10 \% \mathrm{HCl}(100 \mathrm{~mL})$ for hydrolyzation. The hydrolysate was also extracted with ethyl acetate $(3 \times 300 \mathrm{~mL})$ and the organic phase was discarded. After washing by deionized water, the solution was dried through vacuum drying process and the pale yellow powder (eriodictyol) was obtained.

The eriodictyol ( $3.5 \mathrm{~g}$ ) was weighed into a round-bottom flask and dissolved with anhydrous pyridine. The $\mathrm{I}_{2}(1.2 \mathrm{~g})$ was added for reaction with it. After complete reaction, the anhydrous pyridine was evaporated and the pale yellow residue was obtained through vacuum filtration. Then $100 \mathrm{~mL}$ of absolute ethyl alcohol was employed for dissolution and $0.5 \mathrm{~g}$ of activated carbon was added. It was kept at $70{ }^{\circ} \mathrm{C}$ for $30 \mathrm{~min}$ and the luteolin was acquired by filtration.

\subsection{Synthesis of luteolin-manganese(II) complex}

The luteolin-manganese(II) complex was synthesized according to a reported work with appropriate modification. ${ }^{17}$ Briefly, $4.0 \mathrm{~g}$ of luteolin was weighed and subsequently dissolved in $100 \mathrm{~mL}$ 
of absolute ethyl alcohol. A saturated solution of $\mathrm{Mn}\left(\mathrm{CH}_{3} \mathrm{COO}\right)_{2}$ was added drop wise to the resulting solution (in the molar ratio of luteolin : $\mathrm{Mn}^{2+}=2: 1$ ) under continuous stirring. The $\mathrm{pH}$ of the solution was adjusted to 4 with $1 \mathrm{mmol} \mathrm{L}^{-1}$ of $\mathrm{HCl}$. After complete reaction, the solid was precipitated and obtained through filtration. The solid was washed several times with absolute ethyl alcohol and dried in desiccators over anhydrous $\mathrm{MgSO}_{4}$. The luteolin-manganese(II) complex powder was finally obtained. The proposed synthetic path of the co-ordination complex was illustrated in Fig. 1.

\subsection{Physical measurements}

UV-vis spectra of $0.2 \mathrm{mg} \mathrm{mL}^{-1}$ solutions of the luteolin and the luteolin-manganese(II) complex were obtained in deionized water by a UV-vis double beam spectrophotometer (UV-1601, Shimadzu, Kyoto, Japan) using standard $1.00 \mathrm{~cm}$ quartz cells. IR spectra were recorded with a FT-IR (Vertex 70, Bruker, Rheinstetten, Germany) spectrometer in the range of 500$4000 \mathrm{~cm}^{-1}$. Briefly, the luteolin and the luteolin-manganese(II) complex dried completely were thoroughly mixed with $\mathrm{KBr}$ powder, ground and pressed into a $1 \mathrm{~mm}$ pellet, then the prepared samples were determined by FT-IR spectrometer. The TG-DSC analysis measurement of the luteolin-manganese(II) complex under the $\mathrm{N}_{2}$ atmosphere (flow rate: $10 \mathrm{~mL} \mathrm{~min}^{-1}$ ) was performed by a thermal analyzer (STA 449 F3 Jupiter ${ }^{\circledR}$, Netzsch, Bavaria, Germany), between the temperatures of $25-800{ }^{\circ} \mathrm{C}$ at a heating rate of $10{ }^{\circ} \mathrm{C} \mathrm{min}-1$.

\subsection{Elemental analysis}

A scanning electron microscope (Nano SEM430, Nova ${ }^{\mathrm{TM}}$, Hillsboro, Oregon, USA) equipped with an energy dispersive spectroscopy (EDS, Oxford, UK) was used to perform the elemental analysis of the luteolin-manganese(II) complex.

\subsection{Measurements of antioxidant activity}

2.6.1. Analysis of DPPH radical scavenging activity. The DPPH radical scavenging activity was measured according to the method of Dehghan \& Khoshkam (2012) with some modifications. ${ }^{14}$ Briefly, the luteolin and the luteolin-manganese(II) complex were firstly dissolved in dimethyl sulfoxide (DMSO) to obtain different concentrations $\left(0.2-5.0 \mathrm{mg} \mathrm{mL}{ }^{-1}\right)$. Then $1 \mathrm{~mL}$ of sample solution was added to $2 \mathrm{~mL}$ of DPPH solution ( $1 \mathrm{mmol} \mathrm{L}^{-1}$ in methanol). The reaction mixture was shaken vigorously and then incubated in the dark at room temperature for $30 \mathrm{~min}$. Finally, the absorbance of the resulting solution was measured at $517 \mathrm{~nm}\left(A_{\mathrm{i}}\right)$ by the UV-vis double beam spectrophotometer (UV-1601, Shimadzu, Kyoto, Japan). As a control, the absorbance of blank solution of DPPH was also determined at $517 \mathrm{~nm}\left(A_{0}\right)$. The $\mathrm{DPPH}$ radical scavenging activity was calculated according to the following equation:

DPPH radical scavenging activity $(\%)=\left(\frac{A_{0}-A_{\mathrm{i}}}{A_{0}}\right) \times 100 \%$
2.6.2. Analysis of hydroxyl radical scavenging activity. The hydroxyl radical scavenging activity of the luteolin and the luteolin-manganese(II) complex was determined as described in a previous literature. ${ }^{23}$

\subsection{Evaluation of antimicrobial activity}

Antimicrobial activities of the luteolin and the luteolin-manganese(II) complex were assessed using four kinds of bacteria including Escherichia coli, Staphylococcus aureus, Listeria monocytogenes and Pseudomonas aeruginosa. These bacteria stored at $-80{ }^{\circ} \mathrm{C}$ were inoculated to the LB agar culture-medium and then incubated in a constant temperature incubator at $37^{\circ} \mathrm{C}$ for $18-$ $24 \mathrm{~h}$. The bacterial suspensions were prepared via the incubated bacteria and subsequently stored in a refrigerator at $0-4{ }^{\circ} \mathrm{C}$. Afterwards, $0.2 \mathrm{~mL}$ of bacterial suspension for each bacterium was transferred to a new LB agar culture-medium and the susceptibility paper immersed in sample solution for $12 \mathrm{~h}$ was posted on the medium. Three replicates were performed for each bacterium. Penicillin and the sterile water were used as the positive and negative controls, respectively. All the pre-prepared mediums were finally incubated in a constant temperature incubator at $37{ }^{\circ} \mathrm{C}$ for $18-24 \mathrm{~h}$ and the inhibiting ring was measured by a vernier caliper. Each sample was calculated a minimum of three times, and the mean value was calculated and reported.

\subsection{Measurement of the inhibition rate of luteolin and luteolin-manganese(II) complex on the xanthine oxidase (XOD)}

It has been reported that xanthine can be catalyzed by xanthine oxidase, resulting in the production of uric acid which has a characteristic absorption peak at $290 \mathrm{~nm} \cdot{ }^{24}$ So the enzyme activity in the present work was measured spectrophotometrically by continuously measuring uric acid formation at wavelength of $290 \mathrm{~nm}$ with xanthine as the substrate. ${ }^{25}$ Briefly, a series of assay solutions were prepared by dissolving $2 \mathrm{~mL}$ of xanthine substrate (fixed concentration: $0.6 \mathrm{mmol} \mathrm{L}^{-1}$ ) and $0.2 \mathrm{~mL}$ of various concentrations of luteolin or luteolin-manganese(II) complex in $2.8 \mathrm{~mL}$ of sodium phosphate buffer (0.2 mol L $\mathrm{L}^{-1}, \mathrm{pH}$ 7.5). These assay solutions were shaken vigorously and then incubated for $30 \mathrm{~min}$ in a $25^{\circ} \mathrm{C}$ water-bath. Then the assay was initiated by adding $0.2 \mathrm{~mL}$ of xanthine oxidase solution $\left(0.5 \mathrm{U} \mathrm{mL}^{-1}\right)$. The absorbance at $290 \mathrm{~nm}$ was measured every $30 \mathrm{~s}$. The enzymatic activity was finally calculated according to the equation reported by a previous literature. ${ }^{26}$

In the reaction system, the concentration of xanthine substrate was fixed at $0.6 \mathrm{mmol} \mathrm{\textrm {L } ^ { - 1 }}$. The effects of different concentrations of luteolin or luteolin-manganese(II) complex on the enzymatic activity were determined by changing the concentrations of XOD. The linear equations were then obtained by plotting the reaction velocities $(y)$ and XOD concentrations $(x)$ in the enzymatic reaction. A linear equation through the origin indicates the reversible inhibition, while a straight line which is parallel with the $X$ axis accounts for the irreversible inhibition. To describe the competitive inhibition mechanism, 
the concentration of XOD was fixed at $10 \mu \mathrm{g} \mathrm{mL}^{-1}$, and the kinetics of the enzyme in the presence of luteolin or luteolinmanganese(II) complex were investigated using doublereciprocal Lineweaver-Burk plots. ${ }^{24}$

\subsection{Cytotoxicity assay of HepG2 cell}

HepG2 cells were cultured in RPMI 1640 medium supplemented with $10 \% \mathrm{FBS}, 100 \mu \mathrm{g} \mathrm{mL} \mathrm{m}^{-1}$ streptomycin and 100 units per $\mathrm{mL}$ penicillin at $37^{\circ} \mathrm{C}$ in a humidified atmosphere cultivator with $5 \% \mathrm{CO}_{2}$. The cytotoxicity of luteolin or luteolin-manganese(II) complex on HepG2 cells were assessed by the methylene blue assay. ${ }^{27}$ Briefly, HepG2 cells were seeded with a density of 8000 cells per well on a 96-well microplate in $100 \mu \mathrm{L}$ of culture medium per well and subsequently cultured in an incubator at $37^{\circ} \mathrm{C}$ for $24 \mathrm{~h}$. The medium was then removed and the HepG2 cells were washed with $100 \mu \mathrm{L}$ of sterile PBS. After that, $100 \mu \mathrm{L}$ of the medium containing different concentrations of luteolin or luteolin-manganese(II) complex were added to each well. After another $24 \mathrm{~h}$ of incubation, the medium was removed and the HepG2 cells were stained with $50 \mu \mathrm{L}$ of methylene blue. Afterwards, the staining solution was discarded and the microplate was washed by using deionized water. Then $100 \mu \mathrm{L}$ of elution buffer was added to each well and the plates were shaken for $20 \mathrm{~min}$. Finally, the absorbance was measured at $570 \mathrm{~nm}$ with a microplate reader. A control group without sample was conducted in parallel and the absorbance of which was also determined by the microplate reader. The cytotoxicity was calculated using the following equation:

$$
\text { Cytotoxicity }(\%)=1-\frac{A_{\mathrm{s}}-A_{\mathrm{b}}}{A_{\mathrm{c}}-A_{\mathrm{b}}} \times 100 \%
$$

where $A_{\mathrm{s}}$ and $A_{\mathrm{c}}$ are the absorbance of the sample and the control; $A_{\mathrm{b}}$ is the absorbance of the blank; $\frac{A_{\mathrm{s}}-A_{\mathrm{b}}}{A_{\mathrm{c}}-A_{\mathrm{b}}}$ represents the cell viability.

\subsection{Glucose consumption in HepG2}

The glucose consumption in HepG2 was determined according to the method described in a previous work with some modifications. ${ }^{28}$ Briefly, HepG2 cells were seeded with a density of 5000 cells per well on a 96-well microplate and maintained in the DMEM medium at $37^{\circ} \mathrm{C}$ for $12 \mathrm{~h}$. Then they were washed and replaced with DMEM medium which contains high glucose $\left(10 \mathrm{mmol} \mathrm{L}^{-1}\right)$, FCS (5\%) and insulin $\left(0.5 \mu \mathrm{mol} \mathrm{L}{ }^{-1}\right)$. Afterwards, HepG2 cells were incubated at $37{ }^{\circ} \mathrm{C}$ for $24 \mathrm{~h}$ and then washed with serum-free DMEM high glucose. The cells were treated with luteolin or luteolin-manganese(II) complex with different concentrations (1.6, 3.1, 6.3, 12.5 and $25 \mathrm{mmol} \mathrm{L}^{-1}$ ). The glucose concentrations in cell culture supernatant of each group were determined with glucose assay kit (Jiancheng, Nanjing, China) following the manufacturer's instruction. The medium and metformin $\left(25 \mathrm{mmol} \mathrm{L}^{-1}\right.$ ) were used as the control group and the positive control group, respectively.

\subsection{Statistical analysis}

Results were expressed as mean \pm standard deviation (SD) from three replicates for each assay and the data were analyzed by using SPSS 20.0 software (SPSS Inc., Chicago, IL, USA). The analysis of variance (ANOVA) and Tukey's test were used to compare the differences among various groups and a value of $p$ $<0.05$ was considered to be statistically significant.

\section{Results and discussion}

\subsection{UV-visible spectra analysis}

The UV-visible spectra of luteolin (a) and luteolin-manganese(II) complex (b) in deionized water are depicted in Fig. 2. In the UVvisible region of luteolin, two major absorption peaks, like most flavones and flavonols, were observed at 356 and $264 \mathrm{~nm}$ for band I and band II, respectively. This result was similar to a previous study. ${ }^{18}$ It has been demonstrated that band I was annotated for the absorption of cinnamoyl system of luteolin, while band II was considered to be associated with the absorption involving benzoyl system. ${ }^{17}$ In comparison with absorption spectra of luteolin, a significant change was visualized in that of the luteolin-manganese(II) complex due to the red shift of band I, appeared the absorption peak at $414 \mathrm{~nm}$ (band III). The shift of about $58 \mathrm{~nm}$ after the addition of manganese solution to luteolin confirms that complex formation takes place between manganese and luteolin. Moreover, the displacement of band II was also observed from the absorption peak of $264 \mathrm{~nm}$ (band II) to $268 \mathrm{~nm}$ (band IV).

It can be inferred from the chemical structure of luteolin that the 5-hydroxy-4-oxo system (Fig. 1) may be the first site to be involved in the complexation process due to the acidic nature of the $5-\mathrm{OH}$ group and more suitable location of 4 -CO group. ${ }^{16}$ It has been reported that high delocalization of oxygen electrons of $5-\mathrm{OH}$ group could facilitate the $\pi$ electrons delocalization and a big extended $\pi$ bond system could be formed by the interaction of manganese with 5-OH group of luteolin via electronic redistribution. ${ }^{17,22}$ Thus, the new ring formation in luteolin-manganese(II) complex was caused by the enhanced

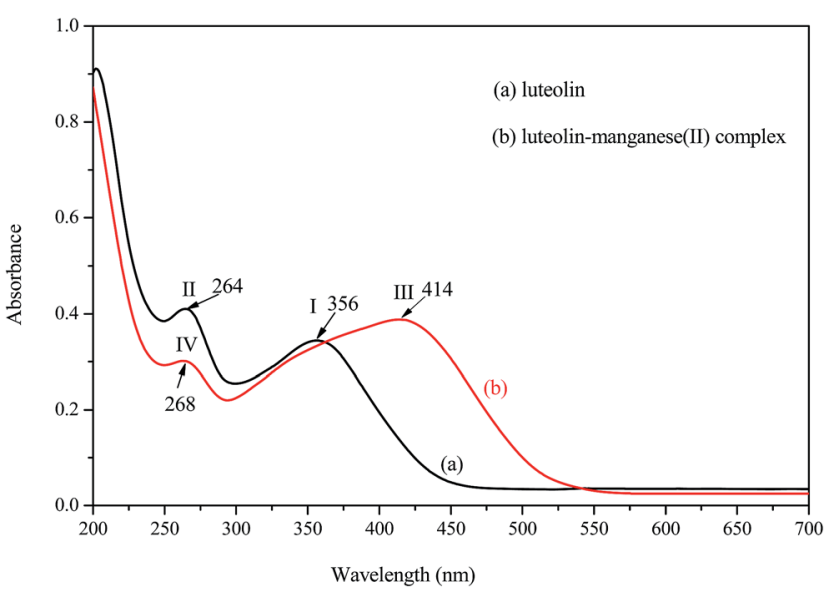

Fig. 2 UV-vis spectra of (a) luteolin and (b) luteolin-manganese(॥) complex. 
conjugative effect with the inclusion of 5-OH and 4-CO groups in the $\mathrm{C}$ ring (Fig. 1). Hence, the bathochromic shift is highly informative for the coordination position in ligand and results obtained also supported the fact that the newly absorption peaks appeared in luteolin-manganese(II) complex were attributable to the presence of $5-\mathrm{OH}$ and $4-\mathrm{CO}$ groups in $\mathrm{A}$ and $\mathrm{C}$ rings.

\subsection{FT-IR spectra analysis}

Fig. 3 depicted FT-IR spectra of luteolin (a) and luteolinmanganese(II) complex (b). The presence of $\nu(\mathrm{Mn}-\mathrm{O})$ stretching vibration peak at $809 \mathrm{~cm}^{-1}$ in the spectrum of luteolin-manganese(II) complex indicated the formation of metal complex via the complexation process, while luteolin exhibited no such band. The characteristic stretching mode of $\mathrm{C}=\mathrm{O}$ was shifted from $1655 \mathrm{~cm}^{-1}$ of luteolin to $1635 \mathrm{~cm}^{-1}$ of luteolin-manganese(II) complex due to the interaction of luteolin with manganese. It can be explained by the co-ordination of carbonyl oxygen with metal ion that confirms to be the manganese(II) had bonded to 5-OH group of $\mathrm{A}$ ring and 4-CO carbonyl group of $\mathrm{C}$ ring. ${ }^{17}$ In addition, a decrease in vibrational frequency of $\nu(\mathrm{C}-\mathrm{OH})$ of luteolin-manganese(II) complex, which was shifted from $1369 \mathrm{~cm}^{-1}$ to $1354 \mathrm{~cm}^{-1}$, was observed probably due to the metal coordination involves the deprotonated ortho-phenolic $(\mathrm{OH})$ group on the A ring of luteolin. ${ }^{18}$ The band at $1164 \mathrm{~cm}^{-1}$ in luteolin which attributes to the $\nu(\mathrm{C}-\mathrm{O}-\mathrm{C})$ stretching vibration mode remained unchanged in luteolin-manganese(II) complex, indicating that the ring oxygen atom was excluded from involvement in the metal coordination. ${ }^{22,29,30}$ The absorption peak at $1655 \mathrm{~cm}^{-1}$ was ascribed to the $\operatorname{ring} \nu(\mathrm{C}=\mathrm{C})$ of luteolin and was shifted to $1585 \mathrm{~cm}^{-1}$ in luteolin-manganese(II) complex. This phenomenon illustrated that a more stable ring was formed due to the increase of conjugate effect after the complex formation. Results discussed above confirmed the complexing reaction of luteolin with manganese(II) and were supportive in the sense that the 5-OH group of A ring and 4-CO carbonyl group of $\mathrm{C}$ ring were the metal complexation site.

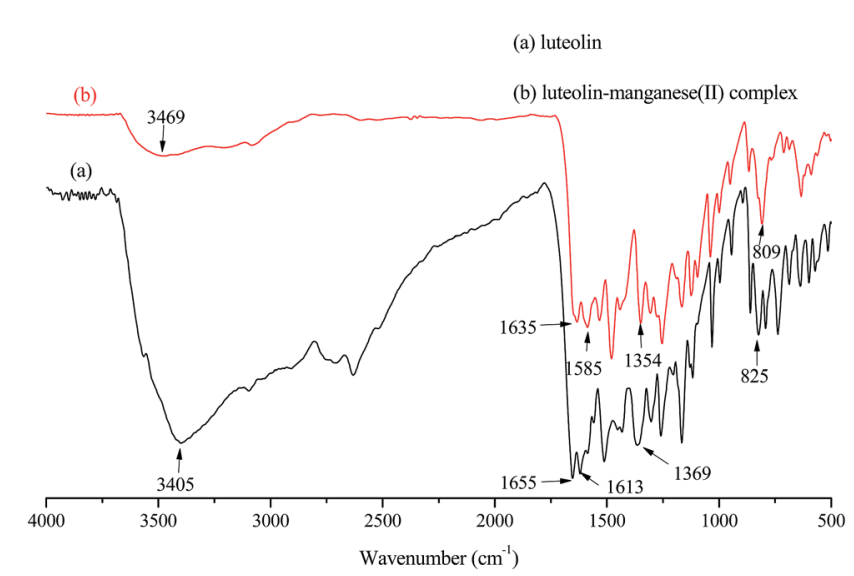

Fig. 3 The IR-spectra of (a) luteolin and (b) luteolin-manganese(॥) complex.

\subsection{Thermal study of the luteolin-manganese(II) complex}

The TG-DSC curves which depict the characteristic thermal events of luteolin-manganese(II) complex were obtained by a thermal analyzer with heating rate of $10{ }^{\circ} \mathrm{C} \mathrm{min}^{-1}$. As shown in Fig. 4, the DSC curve for the complex displayed the endothermic peaks that were completely in agreement with the mass losses observed in the TG curve. The first endothermic peak of luteolin-manganese(II) complex at about $106{ }^{\circ} \mathrm{C}$ observed in the DSC curve probably due to the dehydration of the free moisture ${ }^{18}$ in accordance with $7 \%$ total mass losses in the TG curve. Another endothermic peak around $310{ }^{\circ} \mathrm{C}$ with $20 \%$ total mass losses possibly be related to the melting point of the luteolinmanganese(II) complex or the dehydration of crystal water or coordinated water. ${ }^{18}$ The final thermal decomposition of the luteolin-manganese(II) complex was appeared at above $500{ }^{\circ} \mathrm{C}$ with the final mass losses of $82 \%$.

\subsection{Elemental analysis of the luteolin-manganese(II) complex}

Elemental analysis results of the luteolin-manganese(II) complex are presented in Table 1 and Fig. 5. As shown in Fig. 5, elements of $\mathrm{C}$, $\mathrm{O}$ and $\mathrm{Mn}$ were observed in the luteolin-manganese(II) complex, confirming the coordination reaction between luteolin and the manganese ions. The weight percentages of $\mathrm{C}, \mathrm{O}$ and $\mathrm{Mn}$ in luteolin-manganese(II) complex were $62.48 \%, 29.19 \%$ and $8.33 \%$, respectively, while the corresponding theoretical values were 59.13\%, 31.63\% and 9.06\%, respectively (Table 1). Moreover, the atomic number percentages were $68.24 \%, 28.81 \%$ and $2.95 \%$ respectively, which were also basically consistent with the theoretical values presented in Table 2.

\subsection{Antioxidant activities}

3.5.1. DPPH radical scavenging activity. The DPPH radical is a stable nitrogen-centered free radical which has been widely adopted for evaluating the free radical scavenging activities of naturally antioxidative compounds and antioxidants. ${ }^{32,33}$ The

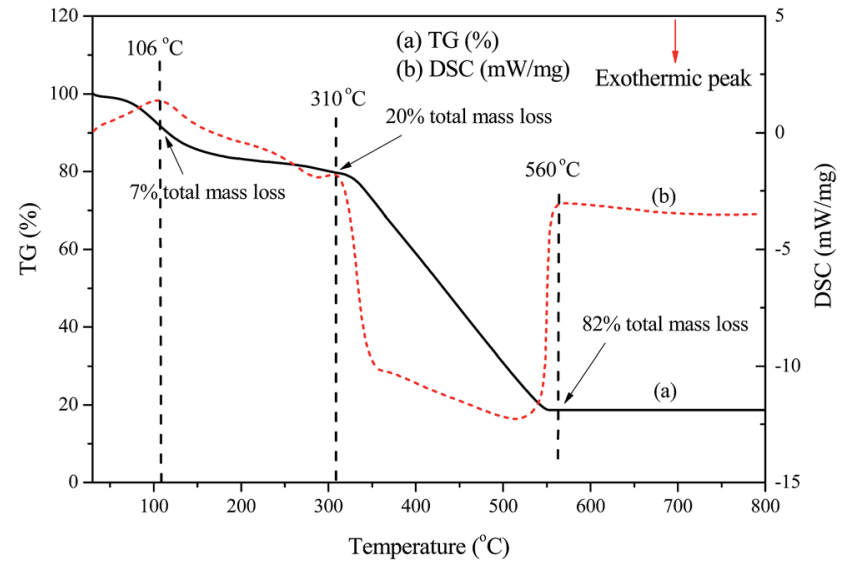

Fig. 4 The TG-DSC curves of the luteolin-manganese(॥) complex with the heating rate of $10{ }^{\circ} \mathrm{C} \mathrm{min}^{-1}$. 
Table 1 Analysis of relative elements mass fraction and relative atoms number of the luteolin-manganese(॥) complex

\begin{tabular}{lclll}
\hline Elements & $\mathrm{Wt}^{a}(\%)$ & $\begin{array}{l}\text { Wt (theoretical } \\
\text { value, \%) }\end{array}$ & $\mathrm{At}^{b}(\%)$ & $\begin{array}{l}\text { At (theoretical } \\
\text { value, \%) }\end{array}$ \\
\hline C & 62.48 & 59.31 & 68.24 & 69.77 \\
O & 29.19 & 31.63 & 28.81 & 27.90 \\
Mn & 8.33 & 9.06 & 2.95 & 2.33
\end{tabular}

${ }^{a}$ Wt: the weight percentage. ${ }^{b}$ At: the atomic number percentage.

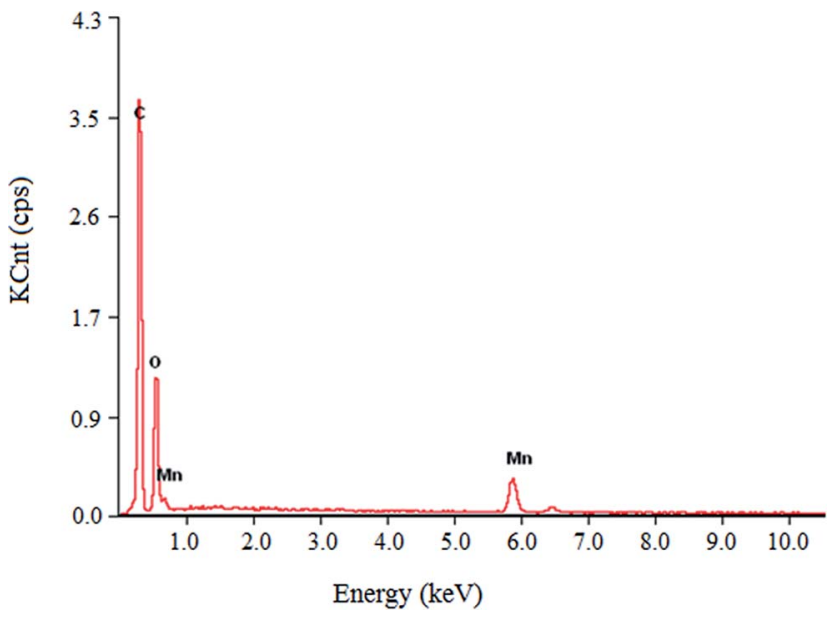

Fig. 5 The elemental composition analysis of luteolin-manganese(॥) complex.

DPPH radical scavenging activities of luteolin and luteolinmanganese(II) complex with different concentrations were monitored and the results are shown in Fig. 6A. Significant increases $(p<0.05)$ of the DPPH radical scavenging activity for both luteolin and luteolin-manganese(II) complex were observed with their concentrations in the range of $0-0.8 \mathrm{mmol} \mathrm{L}^{-1}$. However, the increase of DPPH radical scavenging activity was not significantly $(p>0.05)$ different in the concentrations of $0.8-1.0 \mathrm{mmol} \mathrm{L}^{-1}$. The DPPH radical scavenging activities of $1 \mathrm{mmol} \mathrm{L}^{-1}$ luteolin and $1 \mathrm{mmol} \mathrm{L}^{-1}$ luteolin-manganese(II) complex reached to $75.24 \%$ and $89.14 \%$, respectively. Results confirmed that luteolin was a bioflavonoid with good radical scavenging activity and antioxidant activities. ${ }^{34}$ From Fig. 6A, the DPPH radical scavenging activity of luteolin-manganese(II)
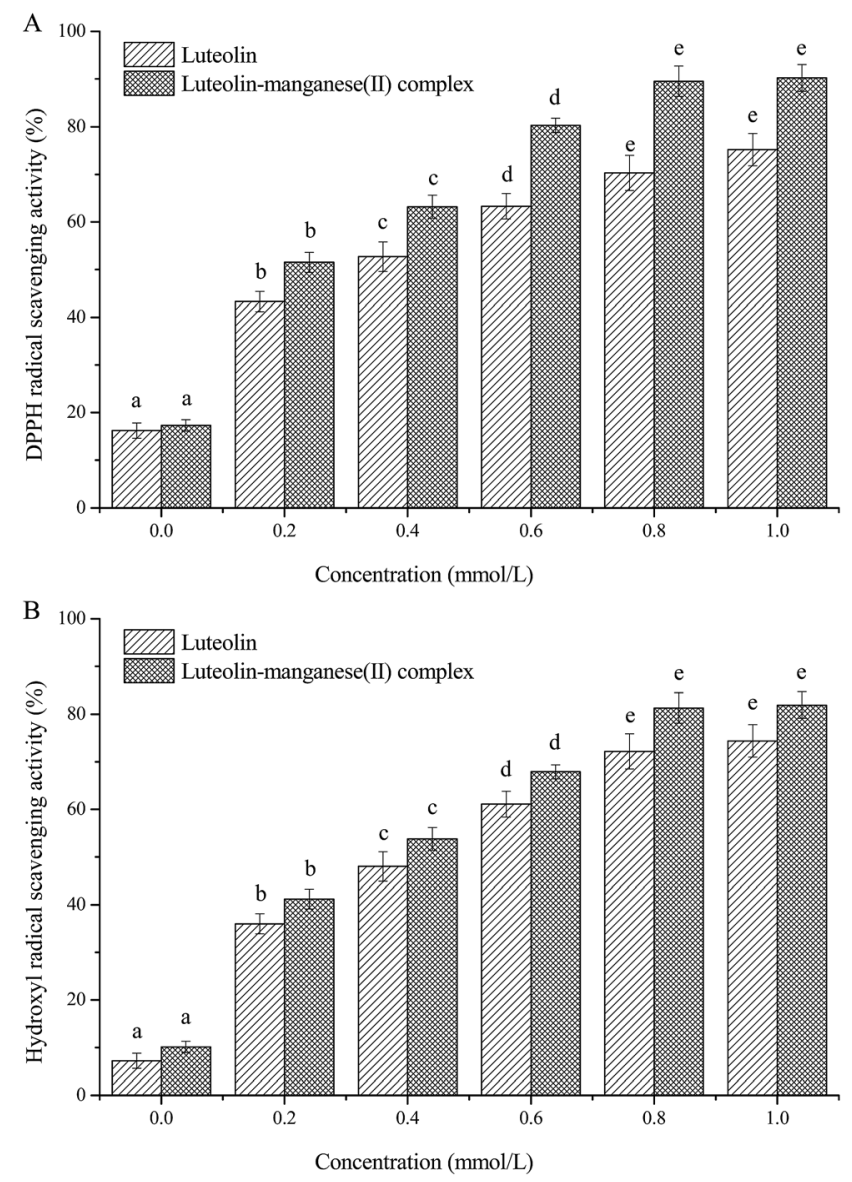

Fig. 6 Effects of luteolin and luteolin-manganese(॥) complex on the $\mathrm{DPPH}$ radical scavenging activity $(\mathrm{A})$ and the hydroxyl radical scavenging activity (B). The data are expressed as means \pm SD with triplicates. Values within the same mullion with different letters above are significantly different at $p<0.01$.

complex was higher than that of luteolin in the same concentrations. In the DPPH radical scavenging reaction, antioxidants donate the hydrogen or electron to $\mathrm{DPPH}^{\circ}$ and convert it into $\mathrm{DPPH}-\mathrm{H}$, thus the antioxidant activity of compounds mainly depends on the electron- or hydrogen-donating ability. ${ }^{35}$ The formation of manganese-luteolin complex electrons increased its scavenging abilities of oxidants and free radicals. ${ }^{36}$ Our findings were also in agreement with a previous work that luteolin-vanadium(II) complex showed a better DPPH radical scavenging ability compared to luteolin. ${ }^{17}$

Table 2 The diameter of inhibition zone of the luteolin and the luteolin-manganese(I) complex ${ }^{a}$

The diameter of inhibition zone $(\mathrm{mm}, n=5)$

\begin{tabular}{lllll} 
Bacteria types & Sterile water & Luteolin & $\begin{array}{l}\text { Luteolin-manganese(II) } \\
\text { complex }\end{array}$ \\
\hline Escherichia coli & $6.02 \pm 0.08^{\mathrm{a}}$ & $7.36 \pm 0.12^{\mathrm{b}}$ & $10.63 \pm 0.18^{\mathrm{c}}$ & Penicillin \\
Staphylococcus aureus & $5.75 \pm 0.09^{\mathrm{a}}$ & $9.56 \pm 0.11^{\mathrm{b}}$ & $12.45 \pm 0.16^{\mathrm{c}}$ & $24.35 \pm 0.16^{\mathrm{d}}$ \\
Listeria monocytogenes & $5.48 \pm 0.06^{\mathrm{a}}$ & $6.78 \pm 0.16^{\mathrm{b}}$ & $9.49 \pm 0.15^{\mathrm{c}}$ & $21.98 \pm 0.21^{\mathrm{d}}$ \\
Pseudomonas aeruginosa & $6.12 \pm 0.08^{\mathrm{a}}$ & $6.89 \pm 0.14^{\mathrm{b}}$ & $8.78 \pm 0.12^{\mathrm{c}}$ & $12.56 \pm 0.12^{\mathrm{d}}$
\end{tabular}

${ }^{a}$ Data are expressed as mean $\pm \mathrm{SD}$ with triplicates. Values within the same row with different letters are significantly different at $p<0.05$. 
3.5.2. Hydroxyl radical scavenging activity. Several diseases such as diabetes is principally induced by hydroxyl radical which has been confirmed to be the most reactive and poisonous free radical. ${ }^{37}$ In order to further verify the antioxidant activity of the prepared complex and its parent compound, luteolin and luteolin-manganese(II) complex of different concentrations were also investigated to scavenge the hydroxyl radical generated from sodium salicylate by the hydroxyl radical scavenging assay. As shown in Fig. 6B, the hydroxyl radical scavenging activities increased significantly $(p<0.05)$ to $74.43 \%$ and $80.34 \%$ respectively after treatment with $1 \mathrm{mmol} \mathrm{L}^{-1}$ luteolin and luteolin-manganese(II) complex. In addition, like the DPPH radical scavenging ability, the hydroxyl radical scavenging activity of luteolin-manganese(II) complex was higher than that of luteolin in the same concentrations, indicating the enhanced antioxidant activity of mental-luteolin complex compared to the parent antioxidant, luteolin. It has been reported that the antioxidant activity of flavonoids mainly depends on their molecular structure, and as for luteolin, it is possibly attributed to the prominent contribution of the $5-\mathrm{OH}$ in A ring. ${ }^{18}$ The significant change of the chemical properties of the ligand luteolin via the chelation reaction with manganese(II) ions is considered to be the main reason for the enhanced antioxidant activity of the complex. ${ }^{38}$

\subsection{Antibacterial activity}

Table 2 describes the antibacterial activities of the luteolin and the luteolin-manganese(II) complex. The diameter of inhibiting zone on the LB agar culture-medium was adopted to evaluate the antibacterial activity. As shown in Table 2, all four bacterial strains treated with luteolin and luteolin-manganese(II) complex presented bigger $(p<0.05)$ diameter of inhibiting zone than the negative control, which were supportive for the obvious antibacterial activity of luteolin and luteolin-manganese(II) complex. Moreover, the bigger $(p<0.05)$ inhibiting rings of luteolin-manganese(II) complex on the inoculated mediums in comparison with those of luteolin verified the enhanced antibacterial activity after the complexation of mental ions with luteolin.

\subsection{Inhibition effects of luteolin and luteolin-manganese(II) complex on XOD}

The inhibition effects of luteolin and luteolin-manganese(II) complex on XOD are displayed in Fig. 7. Sharp increases $(p<$ 0.01) of inhibitory rates to XOD were observed for both luteolin and luteolin-manganese(II) complex with the increased concentrations from 0 to $60 \mu \mathrm{g} \mathrm{mL}{ }^{-1}$, followed by a gentle increase from 60 to $100 \mu \mathrm{g} \mathrm{mL}^{-1}$. At the same concentration, the inhibitory rates of luteolin-manganese(II) complex on XOD was higher than that of luteolin. Luteolin has been reported to have a significant XOD inhibitory activity in comparison with allopurinol. ${ }^{24}$ The results obtained in the present work showed that luteolin-manganese(II) complex has a higher XO inhibitory activity than that of luteolin, resulting from the complexation reaction of manganese ions with luteolin.

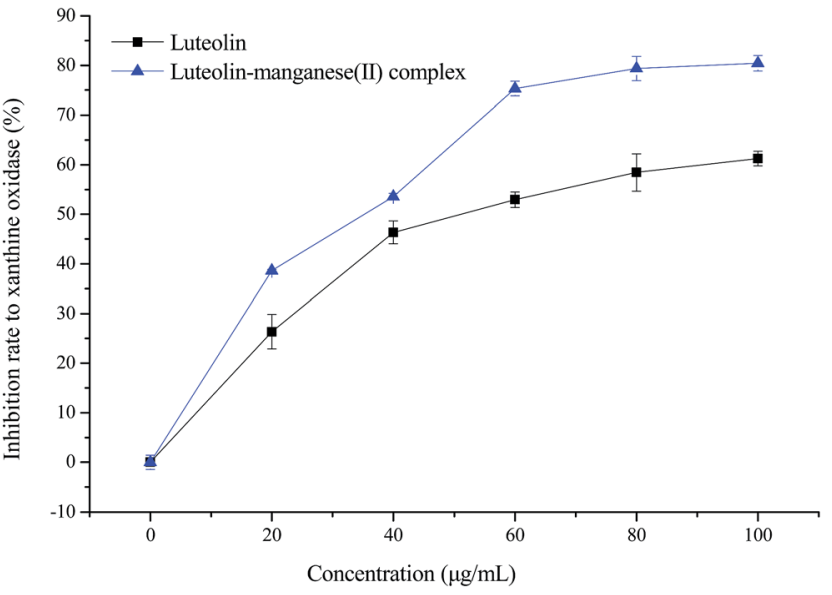

Fig. 7 Inhibitory effect of luteolin and luteolin-manganese(॥) complex on xanthine oxidase. Values are presented as means \pm SD with triplicates.

\subsection{Determining the inhibition type by Lineweaver-Burk plot analysis}

In order to corroborate the reversibility of inhibition, the concentration of xanthine substrate was fixed at $0.6 \mathrm{mmol} \mathrm{L}^{-1}$ and plots of the enzymatic activity $(v) v s$. concentration of XOD were constructed as shown in Fig. 8A and B for luteolin (0, 20, 40, 60 and $\left.80 \mu \mathrm{g} \mathrm{mL}^{-1}\right)$ and luteolin-manganese(II) complex (0, 20, 40, 60 and $80 \mu \mathrm{g} \mathrm{mL}^{-1}$ ), respectively. As depicted in Fig. 8A and B, straight lines for each concentration of luteolin and luteolinmanganese(II) complex were obtained and all passed through the origin. Moreover, the slope of the line was decreased along with the increased concentrations of both luteolin and its complex. Straight lines passing through the origin and decreased slope of the line with increased inhibitor concentrations have been reported to be the obvious phenomenon indicating the reversible inhibition. ${ }^{24}$ Therefore, the inhibition of xanthine oxidase by luteolin and its complex was reversible, which was consistent with the findings investigated in a previous literature. ${ }^{24}$

The kinetics of the xanthine oxidase $\left(10 \mu \mathrm{g} \mathrm{mL} \mathrm{m}^{-1}\right)$ in the presence of luteolin and luteolin-manganese(II) complex were investigated by using double-reciprocal Lineweaver-Burk plots (Fig. 8C and D). It could be clearly observed from Fig. 8C and D that the intercepts of $Y$ axis $[1 / v]$ remained unchanged and the intercepts of $X$ axis $[1 / S]$ increased along with increasing concentrations of luteolin and luteolin-manganese(II) complex. These results indicated that both luteolin and luteolin-manganese(II) complex induced competitive inhibition, which were in agreement with the findings reported in previous work. ${ }^{39}$ This is possibly because that luteolin and luteolin-manganese(II) complex can connected directly with the active position of XOD and compete with the xanthine substrate during the catalytic process. ${ }^{24}$

\subsection{Cytotoxicity and glucose consumption assay in HepG2 cell line}

Normal amounts of insulin are inadequate to trigger normal insulin response in liver, skeletal muscle and adipose tissue, also known as insulin resistance, is the principle characteristic 

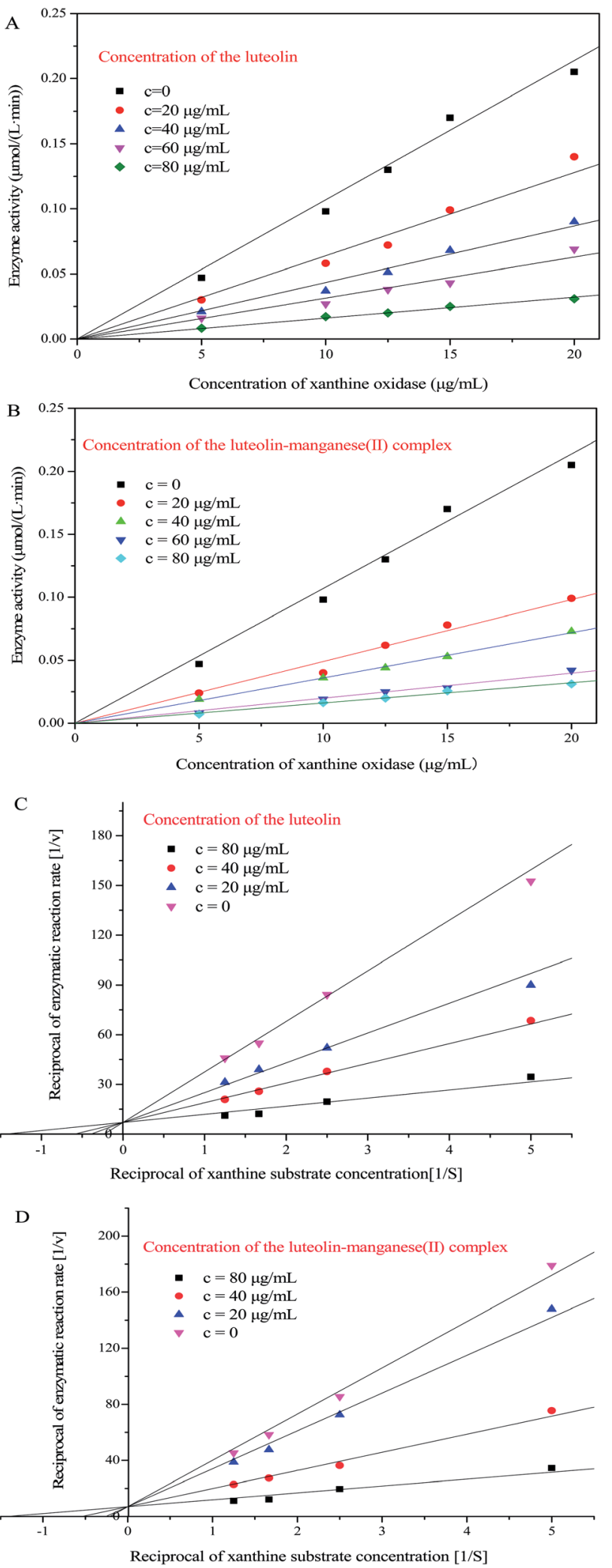

Fig. 8 Plots of enzyme activity $(v)$ vs. concentration of xanthine oxidase (S) for different concentrations of luteolin (A) and luteolinmanganese(॥) complex (B). The concentration of xanthine substrate was $0.6 \mathrm{mmol} \mathrm{L}^{-1}$. Lineweaver-Burk plots of $(1 / \mathrm{v}) \mathrm{vs}$. (1/S) for different concentrations of luteolin (C) and luteolin-manganese(॥) complex (D) The concentration of xanthine oxidase was $10 \mu \mathrm{g} \mathrm{mL}^{-1}$. of diabetes mellitus. ${ }^{40,41}$ It has been proven that skeletal muscle was the main site of insulin-stimulated glucose uptake in vivo and impaired skeletal muscle glucose was the dominating reason leading to the increase of blood glucose in the pathogenesis of insulin resistance and type two diabetes, as glucose transport is the rate-limiting step in muscle glucose metabolism. ${ }^{\mathbf{2 8 , 4 2 , 4 3}}$ However, liver is the primary organ and liver cells become resistant first for metabolism of glucose, resulting in impaired glycogen synthesis and failure to suppress the production of glucose. ${ }^{44}$ Hence, HepG2 cell lines were employed in the present work to investigate glucose uptake by treatment with luteolin and luteolin-manganese(II) complex, and to elucidate the hypoglycemic effect of them. As shown in Fig. 9A, luteolin and luteolin-manganese(II) had no significant influence on the cell viability of HepG2 in the concentration range of 3.1-25 $\mu \mathrm{g} \mathrm{mL} \mathrm{mL}^{-1}$. Several metal-flavonoid complexes were investigated previously ${ }^{\mathbf{3 1 4 5 - 4 7}}$ and some complexes including the vanadium(II)-flavonoid and oxidovanadium(Iv)-flavonoid complexes have been proven to have considerable cytotoxicity on cancer cells. ${ }^{17,22,46,47}$
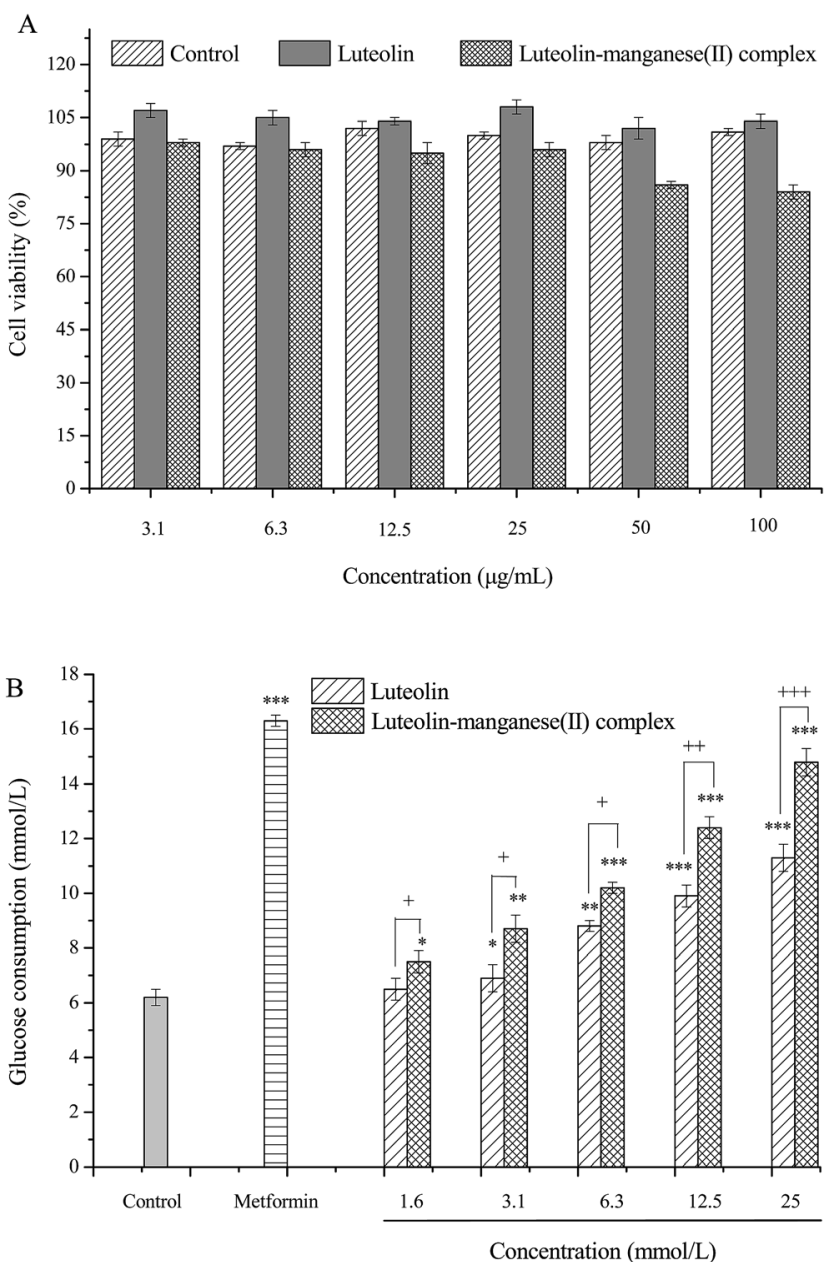

Fig. 9 Effects of luteolin and luteolin-manganese(॥) complex on cell viability (A) and glucose consumption (B) of HepG2 cells. Values are presented as means \pm SD with triplicates. *,** and *** represents $p<$ $0.05, p<0.01$ and $p<0.001$ respectively compared with the control, $+p<0.05,++p<0.01$ and $+++p<0.001$ by Tukey's test. 
After treated with different concentrations of luteolin and luteolin-manganese(II) complex, the glucose consumption by HepG2 cells increased remarkably compared with the control. In particular, the glucose consumption showed a highly significant increase $(p<0.001)$ with the concentrations higher

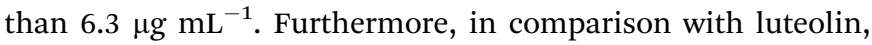
glucose uptake by HepG2 cells treated with luteolin-manganese(II) complex were significant increased at the same concentrations of 1.6, 3.1 and $6.3 \mu \mathrm{g} \mathrm{mL}^{-1}(p<0.05)$, $12.5 \mu \mathrm{g} \mathrm{mL} \mathrm{m}^{-1}(p<0.01)$ and $25 \mu \mathrm{g} \mathrm{mL}{ }^{-1}(p<0.001)$, separately. Results of this test showed that hypoglycemic effects of luteolin and luteolin-manganese(II) complex were obviously achieved by increasing the glucose consumption in liver tissue and luteolinmanganese(II) complex had a more remarkable hypoglycemic effect.

\section{Conclusions}

The metal complex of manganese(II) with luteolin was synthesized and characterized by means of the UV-visible spectroscopy, infrared spectroscopy, thermogravimetric analysis and elemental analysis. Results assumed that the -CO group of the ligand was coordinated with one of the neighboring - $\mathrm{OH}$ group in luteolin. Luteolin was suggested to react with manganese(II) cation through the chelation sites of 5-hydroxy and 4-carbonyl in two luteolin molecules from the spectroscopic data. The luteolin-manganese(II) complex was confirmed to have higher biological activities including antioxidant activity, antibacterial activity and hypoglycemic activity in comparison with the parent luteolin. Luteolin and luteolin-manganese(II) complex reversibly inhibited xanthine oxidase in a competitive manner and the chelation of manganese(II) ions by luteolin showed stronger inhibitory activity on xanthine oxidase than nature luteolin.

\section{Conflicts of interest}

The authors declare that they have no conflicts of interest.

\section{Acknowledgements}

This work was financially supported by the Special Fund Project of Application-oriented for Science and Technology, Research and Development of Guangdong Province (2015B020230001), the Major Special Project of Industry-University-Research Collaborative Innovation of Guangzhou City (201508020086) and the Project of Technology Research Center for Lingnan Characteristic Fruits \& Vegetables Processing and Application Engineering of Guangdong Province (No. [2015]1487), the Project of Food Science Innovation Team of Guangdong Higher Education Institutes (2016KCXTD020).

\section{References}

1 Y. Fu, Y. Zu, W. Liu, T. Efferth, N. Zhang, X. Liu and Y. Kong, Optimization of luteolin separation from pigeonpea
[Cajanus cajan (L.) Millsp.] leaves by macroporous resins, $J$. Chromatogr. A, 2006, 1137, 145-152.

2 A. Kure, K. Nakagawa, M. Kondo, S. Kato, F. Kimura, A. Watanabe, N. Shoji, S. Hatanaka, T. Tsushida and T. Miyazawa, Metabolic Fate of Luteolin in Rats: Its Relationship to Anti-inflammatory Effect, J. Agric. Food Chem., 2016, 64, 4246-4254.

3 P.-C. Lv, H.-Q. Li, J.-Y. Xue, L. Shi and H.-L. Zhu, Synthesis and biological evaluation of novel luteolin derivatives as antibacterial agents, Eur. J. Med. Chem., 2009, 44, 908-914.

4 G. Meng, K. Chai, X. Li, Y. Zhu and W. Huang, Luteolin exerts pro-apoptotic effect and anti-migration effects on A549 lung adenocarcinoma cells through the activation of MEK/ERK signaling pathway, Chem.-Biol. Interact., 2016, 257, 26-34.

5 V. P. Androutsopoulos and D. A. Spandidos, The flavonoids diosmetin and luteolin exert synergistic cytostatic effects in human hepatoma HepG2 cells via CYP1A-catalyzed metabolism, activation of JNK and ERK and P53/P21 upregulation, J. Nutr. Biochem., 2013, 24, 496-504.

6 H. J. Kim, W. Lee and J.-M. Yun, Luteolin Inhibits Hyperglycemia-Induced Proinflammatory Cytokine Production and Its Epigenetic Mechanism in Human Monocytes, Phytother. Res., 2014, 28, 1383-1391.

7 V. Francisco, A. Figueirinha, G. Costa, J. Liberal, M. C. Lopes, C. García-Rodríguez, C. F. G. C. Geraldes, M. T. Cruz and M. T. Batista, Chemical characterization and antiinflammatory activity of luteolin glycosides isolated from lemongrass, J. Funct. Foods, 2014, 10, 436-443.

8 D.-f. Lu, L.-j. Yang, F. Wang and G.-l. Zhang, Inhibitory Effect of Luteolin on Estrogen Biosynthesis in Human Ovarian Granulosa Cells by Suppression of Aromatase (CYP19), J. Agric. Food Chem., 2012, 60, 8411-8418.

9 F. Orhan, M. Gulluce, H. Ozkan and L. Alpsoy, Determination of the antigenotoxic potencies of some luteolin derivatives by using a eukaryotic cell system, Saccharomyces cerevisiae, Food Chem., 2013, 141, 366-372.

10 H. Dong and K. Xiao, Modified QuEChERS combined with ultra high performance liquid chromatography tandem mass spectrometry to determine seven biogenic amines in Chinese traditional condiment soy sauce, Food Chem., 2017, 229, 502-508.

$11 \mathrm{H}$. Dong, K. Xiao, Y. Xian and Y. Wu, Authenticity determination of honeys with non-extractable proteins by means of elemental analyzer (EA) and liquid chromatography (LC) coupled to isotope ratio mass spectroscopy (IRMS), Food Chem., 2018, 240, 717-724.

12 T. Hofer, T. Ø. Jørgensen and R. L. Olsen, Comparison of Food Antioxidants and Iron Chelators in Two Cellular Free Radical Assays: Strong Protection by Luteolin, J. Agric. Food Chem., 2014, 62, 8402-8410.

13 L. Rossi, S. Mazzitelli, M. Arciello, C. R. Capo and G. Rotilio, Benefits from Dietary Polyphenols for Brain Aging and Alzheimer's Disease, Neurochem. Res., 2008, 33, 2390-2400.

14 G. Dehghan and Z. Khoshkam, Tin(II)-quercetin complex: Synthesis, spectral characterisation and antioxidant activity, Food Chem., 2012, 131, 422-426. 
15 V. Uivarosi, S. F. Barbuceanu, V. Aldea, C.-C. Arama, M. Badea, R. Olar and D. Marinescu, Synthesis, Spectral and Thermal Studies of New Rutin Vanadyl Complexes, Molecules, 2010, 15, 1578.

16 R. F. V. de Souza and W. F. De Giovani, Antioxidant properties of complexes of flavonoids with metal ions, Redox Rep., 2004, 9, 97-104.

17 S. Roy, S. Mallick, T. Chakraborty, N. Ghosh, A. K. Singh, S. Manna and S. Majumdar, Synthesis, characterisation and antioxidant activity of luteolin-vanadium(II) complex, Food Chem., 2015, 173, 1172-1178.

18 S. B. Bukhari, S. Memon, M. Mahroof-Tahir and M. I. Bhanger, Synthesis, characterization and antioxidant activity copper-quercetin complex, Spectrochim. Acta, Part A, 2009, 71, 1901-1906.

19 S. M. Ahmadi, G. Dehghan, M. A. Hosseinpourfeizi, J. E. N. Dolatabadi and S. Kashanian, Preparation, Characterization, and DNA Binding Studies of WaterSoluble Quercetin-Molybdenum(VI) Complex, DNA Cell Biol., 2011, 30, 517-523.

20 M. Leopoldini, N. Russo, S. Chiodo and M. Toscano, Iron Chelation by the Powerful Antioxidant Flavonoid Quercetin, J. Agric. Food Chem., 2006, 54, 6343-6351.

21 W. Chen, S. Sun, W. Cao, Y. Liang and J. Song, Antioxidant property of quercetin-Cr(III) complex: the role of $\mathrm{Cr}(\mathrm{III})$ ion, J. Mol. Struct., 2009, 918, 194-197.

22 L. G. Naso, L. Lezama, M. Valcarcel, C. Salado, P. Villacé, D. Kortazar, E. G. Ferrer and P. A. M. Williams, Bovine serum albumin binding, antioxidant and anticancer properties of an oxidovanadium(IV) complex with luteolin, J. Inorg. Biochem., 2016, 157, 80-93.

23 N. Smirnoff and Q. J. Cumbes, Hydroxyl radical scavenging activity of compatible solutes, Phytochemistry, 1989, 28, 1057-1060.

24 J. Yan, G. Zhang, Y. Hu and Y. Ma, Effect of luteolin on xanthine oxidase: inhibition kinetics and interaction mechanism merging with docking simulation, Food Chem., 2013, 141, 3766-3773.

25 U. Takahama, Y. Koga, S. Hirota and R. Yamauchi, Inhibition of xanthine oxidase activity by an oxathiolanone derivative of quercetin, Food Chem., 2011, 126, 1808-1811.

26 L. Lin, Y. Dong, H. Zhao, L. Wen, B. Yang and M. Zhao, Comparative evaluation of rosmarinic acid, methyl rosmarinate and pedalitin isolated from Rabdosia serra (MAXIM.) HARA as inhibitors of tyrosinase and $\alpha$ glucosidase, Food Chem., 2011, 129, 884-889.

27 D. L. Felice, J. Sun and R. H. Liu, A modified methylene blue assay for accurate cell counting, J. Funct. Foods, 2009, 1, 109118.

28 J. Zhu, W. Liu, J. Yu, S. Zou, J. Wang, W. Yao and X. Gao, Characterization and hypoglycemic effect of a polysaccharide extracted from the fruit of Lycium barbarum L, Carbohydr. Polym., 2013, 98, 8-16.

29 L. Naso, E. G. Ferrer, L. Lezama, T. Rojo, S. B. Etcheverry and P. Williams, Role of oxidative stress in the antitumoral action of a new vanadyl(IV) complex with the flavonoid chrysin in two osteoblast cell lines: relationship with the radical scavenger activity, JBIC, J. Biol. Inorg. Chem., 2010, 15, 889-902.

30 E. G. Ferrer, M. V. Salinas, M. J. Correa, L. Naso, D. A. Barrio, S. B. Etcheverry, L. Lezama, T. Rojo and P. A. M. Williams, Synthesis, characterization, antitumoral and osteogenic activities of quercetin vanadyl(IV) complexes, JBIC, J. Biol. Inorg. Chem., 2006, 11, 791-801.

31 J. Li, L. Wang, H. Bai, B. Yang and H. Yang, Synthesis, characterization, and anti-inflammatory activities of rare earth metal complexes of luteolin, Med. Chem. Res., 2011, 20, 88-92.

32 C. Li, Q. Huang, X. Fu, X.-J. Yue, R. H. Liu and L.-J. You, Characterization, antioxidant and immunomodulatory activities of polysaccharides from Prunella vulgaris Linn, Int. J. Biol. Macromol., 2015, 75, 298-305.

33 C. Li, X. Fu, Q. Huang, F. Luo and L. You, Ultrasonic extraction and structural identification of polysaccharides from Prunella vulgaris and its antioxidant and antiproliferative activities, Eur. Food Res. Technol., 2015, 240, 49-60.

34 M. Lopez-Lazaro, Distribution and Biological Activities of the Flavonoid Luteolin, Mini-Rev. Med. Chem., 2009, 9, 31-59.

35 K. Ghafoor, Y. H. Choi, J. Y. Jeon and I. H. Jo, Optimization of Ultrasound-Assisted Extraction of Phenolic Compounds, Antioxidants, and Anthocyanins from Grape (Vitis vinifera) Seeds, J. Agric. Food Chem., 2009, 57, 4988-4994.

36 Y. Li, B. Jiang, T. Zhang, W. Mu and J. Liu, Antioxidant and free radical-scavenging activities of chickpea protein hydrolysate (CPH), Food Chem., 2008, 106, 444-450.

37 J.-E. Li, S.-P. Nie, M.-Y. Xie and C. Li, Isolation and partial characterization of a neutral polysaccharide from Mosla chinensis Maxim. cv. Jiangxiangru and its antioxidant and immunomodulatory activities, J. Funct. Foods, 2014, 6, 410418.

38 H. J. Lee, R. A. Kerr, K. J. Korshavn, J. Lee, J. Kang, A. Ramamoorthy, B. T. Ruotolo and M. H. Lim, Effects of hydroxyl group variations on a flavonoid backbone toward modulation of metal-free and metal-induced amyloid[small beta] aggregation, Inorg. Chem. Front., 2016, 3, 381392.

39 H.-C. Lin, S.-H. Tsai, C.-S. Chen, Y.-C. Chang, C.-M. Lee, Z.-Y. Lai and C.-M. Lin, Structure-activity relationship of coumarin derivatives on xanthine oxidase-inhibiting and free radical-scavenging activities, Biochem. Pharmacol., 2008, 75, 1416-1425.

40 X. Zhou, J. Chen, F. Wang, H. Yang, R. Yang, X. Wang and Y. Wang, Selenium-enriched exopolysaccharides improve skeletal muscle glucose uptake of diabetic KKAy mice via AMPK pathway, J. Physiol. Biochem., 2014, 70, 547-554.

41 C. Chen, B. Zhang, X. Fu and R. H. Liu, A novel polysaccharide isolated from mulberry fruits (Murus alba L.) and its selenide derivative: structural characterization and biological activities, Food Funct., 2016, 7, 2886-2897.

42 W. Xie, W. Wang, H. Su, D. Xing, Y. Pan and L. Du, Effect of ethanolic extracts of Ananas comosus L. leaves on insulin 
sensitivity in rats and HepG2, Comp. Biochem. Physiol., Part C: Toxicol. Pharmacol., 2006, 143, 429-435.

43 Y. Sogame, A. Kitamura, M. Yabuki and S. Komuro, Liver uptake of Biguanides in rats, Biomed. Pharmacother., 2011, 65, 451-455.

$44 \mathrm{~J} . \mathrm{Xu}, \mathrm{M}$. Ma and W. M. Purcell, Characterisation of some cytotoxic endpoints using rat liver and HepG2 spheroids as in vitro models and their application in hepatotoxicity studies. I. Glucose metabolism and enzyme release as cytotoxic markers, Toxicol. Appl. Pharmacol., 2003, 189, 100-111.
45 N. Muhammad and Z. Guo, Metal-based anticancer chemotherapeutic agents, Curr. Opin. Chem. Biol., 2014, 19, 144-153.

46 B. Biersack, A. Ahmad, F. H. Sarkar and R. Schobert, Coinage Metal Complexes Against Breast Cancer, Curr. Med. Chem., 2012, 19, 3949-3956.

47 H. Dong, K. Xiao, D. Luo, Y. Xian, H. Luo, X. Guo, C. Li and M. Zhao, Adulteration identification of commercial honey with the C-4 sugar content of negative values by an elemental analyzer and liquid chromatography coupled to isotope ratio mass spectroscopy, J. Agric. Food Chem., 2016, 64, 3258-3265. 